\title{
Rapid Microwave-Only Characterization and Readout of Quantum Dots Using Multiplexed Gigahertz-Frequency Resonators
}

\author{
Damaz de Jong $\odot,{ }^{1, *}$ Christian G. Prosko $\odot,{ }^{1}$ Daan M. A. Waardenburg, ${ }^{1}$ Lin Han,${ }^{1}$ \\ Filip K. Malinowski® ${ }^{1}{ }^{\text {Peter Krogstrup, }}{ }^{2}$ Leo P. Kouwenhoven, ${ }^{1,3}$ Jonne V. Koski, ${ }^{3}$ and \\ Wolfgang Pfaffo ${ }^{3,4, \dagger}$ \\ ${ }^{1}$ QuTech and Kavli Institute of Nanoscience, Delft University of Technology, 2600 GA Delft, The Netherlands \\ ${ }^{2}$ Center for Quantum Devices, Niels Bohr Institute, University of Copenhagen \& Microsoft Quantum Materials \\ Lab, Copenhagen, Denmark \\ ${ }^{3}$ Microsoft Quantum Lab Delft, Delft University of Technology, 2600 GA Delft, The Netherlands \\ ${ }^{4}$ Department of Physics and Frederick Seitz Materials Research Laboratory, University of Illinois at \\ Urbana-Champaign, Urbana, Illinois 61801, USA
}

(Received 9 March 2021; accepted 19 May 2021; published 2 July 2021)

\begin{abstract}
Superconducting resonators enable fast characterization and readout of mesoscopic quantum devices. Finding ways to perform measurements of interest on such devices using resonators only is therefore of great practical relevance. We report an experimental investigation of an InAs nanowire multiquantum dot device by probing gigahertz resonators connected to the device. First, we demonstrate accurate extraction of the dc conductance from measurements of the high-frequency admittance. Because our technique does not rely on dc calibration, it could potentially obviate the need for dc measurements in semiconductor qubit devices. Second, we demonstrate multiplexed gate sensing and the detection of charge tunneling on microsecond timescales. The gigahertz detection of dispersive resonator shifts allows rapid acquisition of charge stability diagrams, as well as resolving charge tunneling in the device with a signal-to-noise ratio of up to 15 in $1 \mu \mathrm{s}$. Our measurements show that gigahertz-frequency resonators may serve as a universal tool for fast tuneup and high-fidelity readout of semiconductor qubits.
\end{abstract}

DOI: 10.1103/PhysRevApplied.16.014007

\section{INTRODUCTION}

Microwave resonators in the few-gigahertz range are well known as a powerful means to increase the speed with which properties of mesoscopic quantum devices can be read out [1]. In the field of quantum information, resonators in this so-called superhigh-frequency (SHF) band have thus enabled the fast and high-fidelity nondemolition readout of quantum bits (qubits)[2-5], as well as mediating interactions between qubits [6-9]. SHF resonators are also an attractive tool for the fast characterization of quantum devices, because the required tuneup routines are generally time-consuming. Additionally, frequency multiplexing using many high-Q resonators has been established for hardware-efficient mass-characterization of devices $[10,11]$.

\footnotetext{
*damazdejong@gmail.com

†wpfaff@illinois.edu
}

Published by the American Physical Society under the terms of the Creative Commons Attribution 4.0 International license. Further distribution of this work must maintain attribution to the author(s) and the published article's title, journal citation, and DOI.
Efficient characterization is particularly relevant for semiconductor quantum devices where many gate electrodes result in a large parameter space. In recent years there have been numerous efforts to utilize SHF resonators for this purpose [12-16] as well as reading out qubit degrees of freedom [17-26]. Despite these successes, however, experiments are still often supplemented with dc or low-frequency measurements to quantitatively extract the dc conductance [27]. As larger-scale devices are developed [28,29], it is interesting to direct focus to readout and tuneup schemes utilizing SHF resonators only, thus allowing a single framework for all measurements performed on a device.

Here, we present experiments using multiplexed resonators in the range 3-7 GHz coupled to a multiquantum dot (multi-QD) system. Using the resonator response only, we are able to infer quantitatively the dc conductance of the system, and detect single-electron tunneling with high signal-to-noise ratio (SNR) on submicrosecond timescales. The remainder of this paper is organized as follows. In Sec. III, we determine the dc (i.e., zero-frequency) conductance from SHF measurements without any dc calibration data and find agreement with conductance obtained from a dc transport control measurement. In Sec. IV, 
we demonstrate fast multiplexed dispersive gate sensing (DGS) at gigahertz frequencies in a double quantum dot (DQD). This local measurement of charge transitions facilitates fast tuneup of multi-QD systems [29]. Finally, in Sec. V, we attain high SNRs in the detection of charge tunneling in the DQD. State-dependent charge tunneling is a key mechanism for qubit readout in semiconductor qubits [30]. Our optimized resonator design [31], combined with the use of a near-quantum-limited amplifier [32], results in a maximum SNR of 15 in an integration time of $1 \mu \mathrm{s}$.

\section{EXPERIMENTAL SETUP}

The device comprises an InAs nanowire with a gigahertz-bandwidth coplanar waveguide resonator [33] coupled to every QD to sense the electronic compressibility of each individual dot. An additional resonator that is galvanically connected to the source of the nanowire is used to probe the admittance of the nanowire. Figures 1(a) and 1(b) show images of the resonators and the multiQD device, respectively. An approximate lumped-element schematic of the device is shown in Fig. 1(c). Each resonator is coupled to a central feedline in a hanger
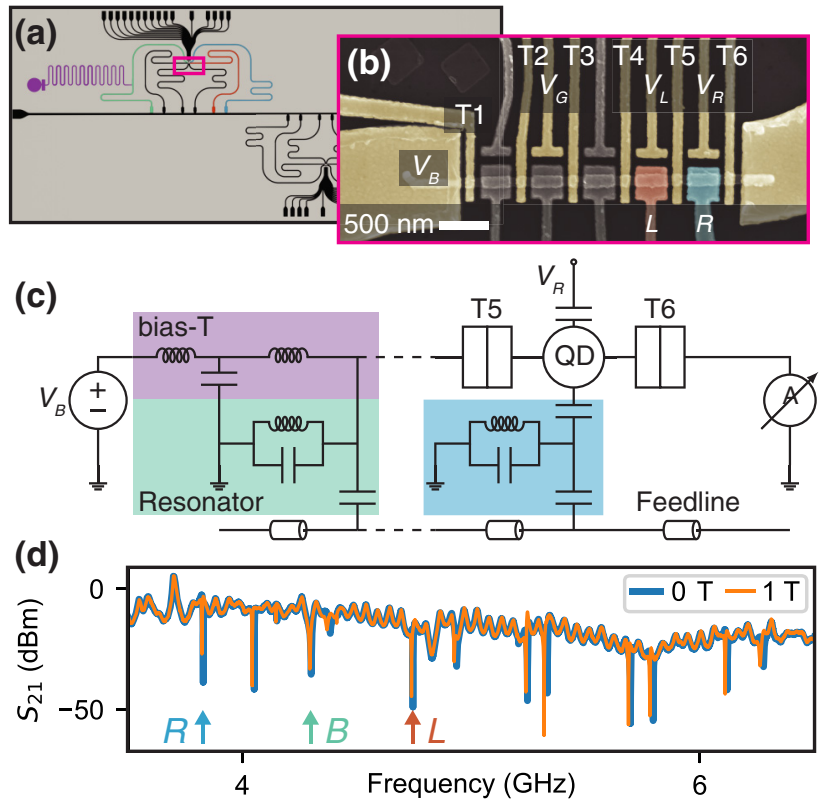

FIG. 1. Experimental setup and resonator response. (a) Schematic of the device layout. (b) False-colored electron micrograph of the nanowire and the surrounding gates. (c) The rf equivalent circuit diagram of the device. The five topgates are coupled to resonators as is the source electrode of the nanowire that can be dc-biased by $V_{B}$ with a bias-T. The topgates are separated by six tunnel gates such that the nanowire can be pinched off at various positions and quantum dots can be defined. The charge on the quantum dots can be controlled by the sidegates. (d) Transmission through the feedline without magnetic field and at $1 \mathrm{~T}$ applied parallel to the plane of the resonators. The arrows $L$ (left), $R$ (right), and $B$ (bias) mark the resonators used here. geometry and is individually addressable using frequency multiplexing [Fig. 1(d)]. The obtained SNR is set by the high resonator bandwidth, optimized resonator coupling quality factors, and a traveling-wave parametric amplifier (TWPA) [32] at the base temperature stage of $20 \mathrm{mK}$ of our dilution refrigerator. For further details, see the Supplemental Material [34].

\section{HIGH-FREQUENCY CONDUCTANCE MEASUREMENTS}

We begin by investigating the SHF response of the resonator coupled to the lead in response to changing nanowire conductance [12-14,27,35,36]. By tuning the gate voltage $\mathrm{T} 2$ and keeping the other gates at $0 \mathrm{~V}$ we alter the nanowire conductance. This modulates the resonator response, shown in Figs. 2(a) and 2(b), through changes in its load admittance. The dc conductance can be extracted from the load admittance either by building up a calibration map of load admittance and dc conductance or by quantitatively modeling the resonator circuit [27]. We take the latter approach to maintain independence from dc calibration measurements. To quantify the modulation of the resonator response, we fit the response to a hanger input-output model [37-39]. The relevant parameters for extracting load admittance are the change in the resonance frequency $\Delta \omega_{0}$ and the additional photon decay rate $\Delta \kappa_{d}$ with respect to the pinchedoff regime, which is reached by decreasing the gate voltages until $\kappa_{d}$ saturates. Representative fits are plotted in Fig. 2(a) and the extracted $\kappa_{d}$ and $\Delta \omega_{0}$ are shown in Fig. 2(c). The load admittance, $Y$, can then be calculated by

$$
Y=\frac{\pi}{Z_{0} \omega_{0}}\left(\frac{1}{2} \Delta \kappa_{d}-\imath \Delta \omega_{0}\right)
$$

which holds for a transmission line resonator of characteristic impedance $Z_{0}$ coupled to a high impedance load $1 /|Y| \gg Z_{0}$. We estimate $Z_{0}=116 \Omega$ from the resonator design. See the Supplemental Material for more details of the procedure outlined above [34].

Importantly, the load admittance at finite frequency does not directly translate to the dc conductance of the coupled device (i.e., the nanowire). The nanowire itself has an inductive component and the gates surrounding the nanowire add additional shunting capacitive paths to ground, contributing to the load admittance especially for higher frequencies. Our device design with high lever-arm gates necessitates compensating for these contributions explicitly, in contrast to the experiments in Refs. [13,14,27]. To account for these effects, we model the load admittance $Y$ as in Fig. 2(d), describing an effective transmission line formed by the nanowire split by a tunnel junction. We denote the series resistance, inductance, and parallel capacitance per unit length of this transmission 


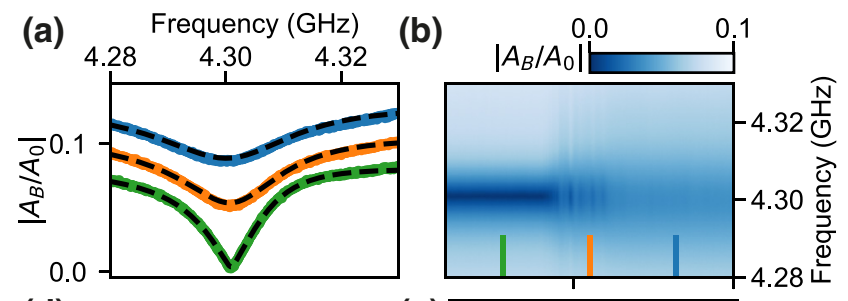

(d)
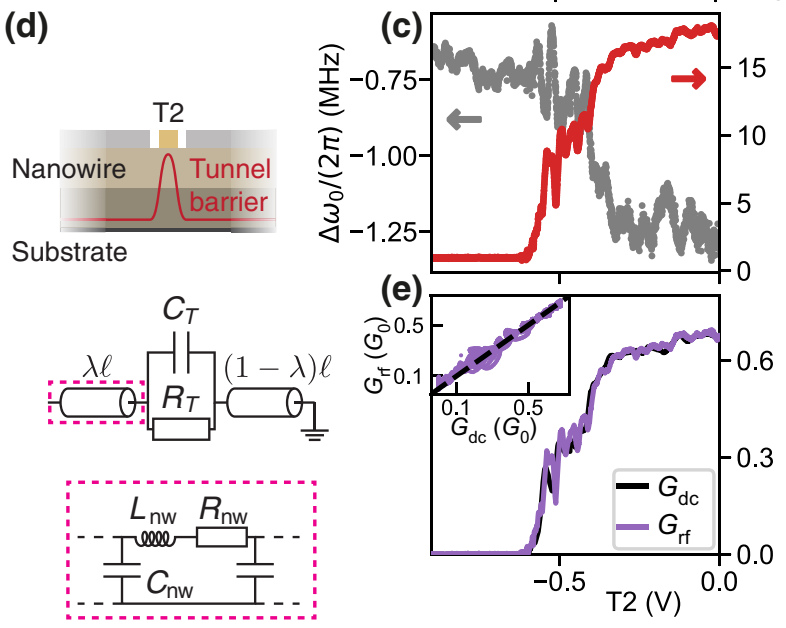

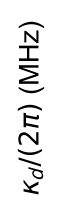

FIG. 2. Pinchoff measurements. (a),(b) Response of the conductance resonator to the tunnel gate voltage T2 and linecuts at the indicated gate voltages in (b) offset for clarity. The quantity $\left|A_{B} / A_{0}\right|$ denotes the ratio of measured signal to input signal. (c) Frequency shift $\Delta \omega_{0}$ and internal resonator decay $\kappa_{d}$ extracted from individual resonator line traces of (b). (d) Schematic of the nanowire for the experiment in (b) with the corresponding lumped-element model used to convert between resonator admittance and conductance $G_{\mathrm{rf}}$. (e) Conductance $G_{\mathrm{dc}}$, measured with standard voltage-biased current measurements, together with the conductance $G_{\text {rf }}$ extracted from (c). The inset shows the conductance $G_{\mathrm{rf}}$ as a function of conductance $G_{\mathrm{dc}}$, for the gate response of all tunnel gate voltages $\mathrm{T} 1$ through $\mathrm{T} 6$. The dashed line indicates $G_{\mathrm{dc}}=G_{\mathrm{rf}}$. The individual traces are included in the Supplemental Material [34]. All measurements in this figure are taken at $V_{B}=10 \mathrm{mV}$ while unused gates are held at $0 \mathrm{~V}$ such that only the active tunnel gate can deplete the nanowire.

line by $R_{\mathrm{nw}}, L_{\mathrm{nw}}$, and $C_{\mathrm{nw}}$ and introduce $Z_{\mathrm{nw}}=\ell\left(R_{\mathrm{nw}}+\right.$ $\left.\imath \omega L_{\mathrm{nw}}\right)$ with $\ell$ the nanowire length. The dc conductance of the nanowire can be calculated from the impedance added by the nanowire itself, $Z_{\mathrm{nw}}$, and the impedance of the tunnel junction $Z_{T}$.

The relation between $Z_{T}$ and $Y$ depends on the fractional position of the tunnel junction along the nanowire, which we parameterize by $\lambda$. Explicitly, the relation is given by

$$
Z_{T}=\frac{\frac{Z_{\mathrm{nw}}}{\gamma \ell}}{\cosh [(1-\lambda) \gamma \ell]} \frac{\frac{Y Z_{\mathrm{nw}}}{\gamma \ell} \sinh (\gamma \ell)-\cosh (\gamma \ell)}{\sinh (\lambda \gamma \ell)-\frac{Y Z_{\mathrm{nw}}}{\gamma \ell} \cosh (\lambda \gamma \ell)},
$$

where $\gamma \equiv \sqrt{\left(R_{\mathrm{nw}}+\imath \omega L_{\mathrm{nw}}\right) \iota \omega C_{\mathrm{nw}}}$ denotes the complex propagation constant.
The constants $Z_{\mathrm{nw}}$ and $\gamma \ell$ are determined from two SHF calibration measurements. For the first calibration measurement, the load impedance $Y_{o}$ is measured when all gates are open at $0 \mathrm{~V}$, corresponding to the limit that $Z_{T}=0$. For the second calibration measurement, the load impedance $Y_{p}$ as $\left|Z_{T}\right| \rightarrow \infty$ and $\lambda=1$ is measured by tuning the rightmost gate voltage T6 into pinchoff. Solving the resulting two equations for $\gamma \ell$ and $Z_{\mathrm{nw}}$ yields

$$
\gamma \ell=\operatorname{arctanh}\left(\sqrt{\frac{Y_{p}}{Y_{o}}}\right) \quad \text { and } \quad Z_{\mathrm{nw}}=\frac{\gamma \ell}{\sqrt{Y_{p} Y_{o}}} .
$$

See the Supplemental Material for more information [34]. Using Eqs. (2) and (3), we then extract $Z_{T}$ from the admittance $Y$. We model the junction as a resistor $R_{T}$ and capacitor $C_{T}$ in parallel such that $Z_{T}^{-1} \equiv 1 / R_{T}+i \omega C_{T}$ [40], and then determine the dc-equivalent conductance as

$$
G_{\mathrm{rf}}^{-1}=\operatorname{Re}\left(Z_{\mathrm{nw}}\right)+1 / \operatorname{Re}\left(Z_{T}^{-1}\right) .
$$

To validate our method to infer the conductance, we compare it with the conductance obtained from a control experiment using conventional de detection. Figure 2(e) shows the conductance extracted from dc measurements $G_{\mathrm{dc}}$ and the dc conductance extracted from the resonator response $G_{\mathrm{rf}}$. Excellent agreement is observed between $G_{\mathrm{rf}}$ and $G_{\mathrm{dc}}$ for data from pinchoff traces of T1 through T6, changing $\lambda$ according to the position of the gate, shown in the inset of Fig. 2(e).

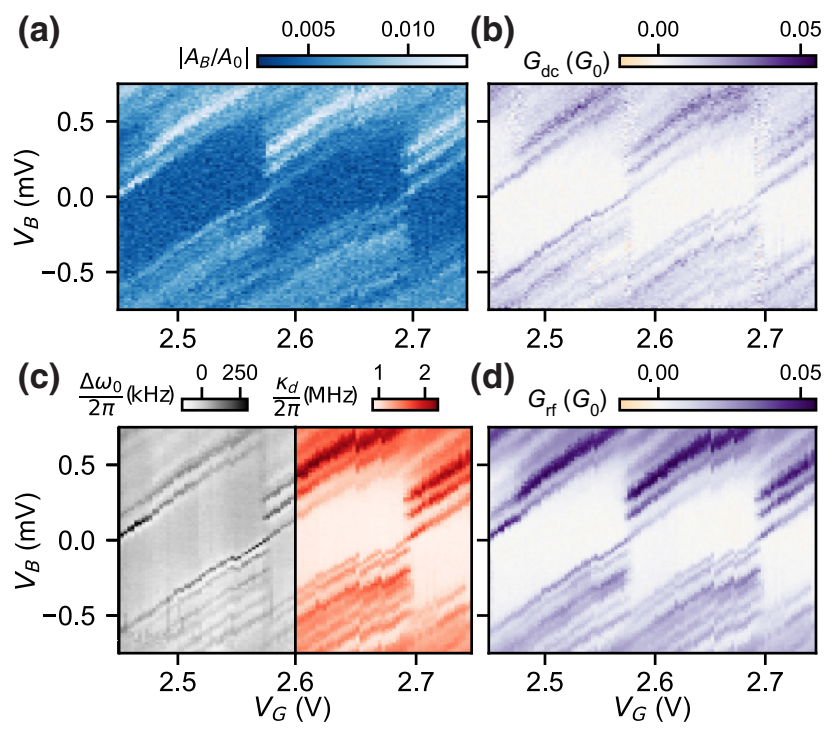

FIG. 3. Coulomb blockade diamonds measured in a single quantum dot. (a) Single-frequency response of the resonator. (b) $G_{\mathrm{dc}}$ measurements obtained with standard lock-in techniques. (c) Frequency shift $\Delta f$ and resonator decay rate $\kappa_{d}$ extracted from frequency traces. (d) Conductance $G_{\mathrm{rf}}$ extracted from resonator data in (c). 
Applications of rf conductance are not limited to measuring the impedance of tunnel gates [16,41-43]. As an example, we probe a quantum dot by tuning $\mathrm{T} 2$ and $\mathrm{T} 3$ into the tunneling regime and modulating the gate voltage $V_{G}$, leaving the other gates at $0 \mathrm{~V}$. We show the amplitude response of the lead resonator on resonance in Fig. 3(a) as a function of bias voltage $V_{B}$ and gate voltage $V_{G}$. Even though the amplitude response is not translated into dc conductance here, it shows all the qualitative features present in the control data measured by dc lock-in conductance [Fig. 3(b)], including the excited states of the quantum dot. The amplitude response of Fig. 3(a) is part of a full frequency trace, measured to also allow for a quantitative comparison between the dc results and the resonator response. From these traces, the frequency shift $\Delta \omega_{0}$ and photon decay $\Delta \kappa_{d}$ are extracted and shown in Fig. 3(c). We use the model defined by Eqs. (1)-(4) to obtain $G_{\mathrm{rf}}$, shown in Fig. 3(d). This is the same model used for the tunnel junction scans of Fig. 2. Note that we neglect here the finite width occupied by the quantum dot and its internal structure; nevertheless we observe reasonable agreement between $G_{\mathrm{rf}}$ and $G_{\mathrm{dc}}$.

\section{RAPID MULTIPLEXED REFLECTOMETRY}

We now move on to the capacitively coupled gate resonators and investigate DGS in the DQD regime $[17,25,44-53]$. To tune the system into a DQD, the gate voltages T4, T5, and T6 are each decreased into the tunneling regime. Accordingly, two quantum dots are formed under the rightmost two topgates in the nanowire [40].

A resonator is coupled to both dots to sense the electronic compressibility of the individual dots [54,55]. In Fig. 4 we show a charge stability diagram (CSD) using $V_{L}$ and $V_{R}$ to change the electron occupation of the DQD. We perform pulsed readout with an integration time of $3 \mu$ s per point, constituting a total data acquisition time of $30 \mathrm{~ms}$ for the entire CSD $[15,56]$. The data acquisition is frequency-multiplexed for both resonators such that the data in Figs. 4(a) and 4(b) are measured simultaneously [29,57]. This not only reduces the measurement time, but multiplexing also guarantees that the measurements in Figs. 4(a) and 4(b) correspond to the exact same physical regime, regardless of charge jumps and gate hysteresis. To emphasize the correspondence between Figs. 4(a) and 4(b), the same guides to the eye outlining stable charge configurations are drawn in both panels.

Resonators are only sensitive to charge transitions involving the quantum dots to which they are coupled. Therefore, both resonators detect the interdot transitions; however, transitions from the right dot to the right electrode are detected only by the resonator connected to the right dot. Here, the resonator connected to the left dot does not respond to transitions between the left dot and the left electrode. We attribute this to a mismatched

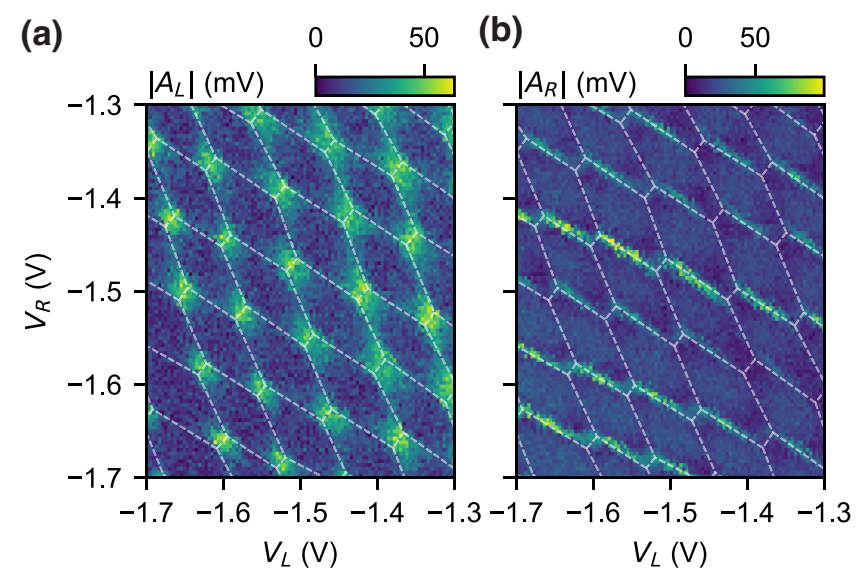

FIG. 4. Charge stability diagram measured using multiplexed gate-based readout in the double dot regime. (a),(b) Amplitude response of the resonators coupled to the two rightmost quantum dots. Readout power in the feedline is $-105 \mathrm{dBm}$ per multiplexed resonator with an integration time of $3 \mu \mathrm{s}$. The dimensions of this dataset are $101 \times 101$ points yielding a total integration time of $30 \mathrm{~ms}$ excluding overhead from gate settling time, set by low-pass filters on the gate wiring. The dashed lines are guides to the eye delineating the different charge configurations of the double dot and are identical in (a) and (b).

left dot-left electrode tunnel coupling. Hence multiplexing also enables spatial correlation of electron tunneling by comparing the DGS signal from each gate's resonator, effectively "tracking" the electron through the device.

\section{SIGNAL-TO-NOISE RATIO}

Finally, we investigate the attainable SNR for resolving charge tunneling with DGS by changing detuning from charge degeneracy in the DQD. This procedure serves as a proxy for different qubit states in schemes where readout is based on state-dependent tunneling [25,36,51,58-60]. Because actual qubit systems will have limitations on the readout power [30] we investigate the SNR both at a fixed "low" excitation voltage in the resonator, $V_{e}=5 \mu \mathrm{V}$, as well as at an optimized excitation voltage, $V_{e}=0.16 \mathrm{mV}$. These excitation voltages are calculated from the generator power and line attenuation in addition to the resonator frequency and coupling capacitance to the feedline.

We fix the total charge in the system by pinching off gates on either side of the DQD. The only remaining transitions are interdot transitions occurring through a tunnel coupling denoted by $t_{C}$. The resonator response as a function of the energy detuning $\delta$ from the interdot transition is shown in Fig. 5(a). We determine $t_{C}$ by fitting the resonator response to an input-output model [18], discussed in the Supplemental Material [34]. Linecuts of the fit results and measurement data are shown in Fig. 5(b). 


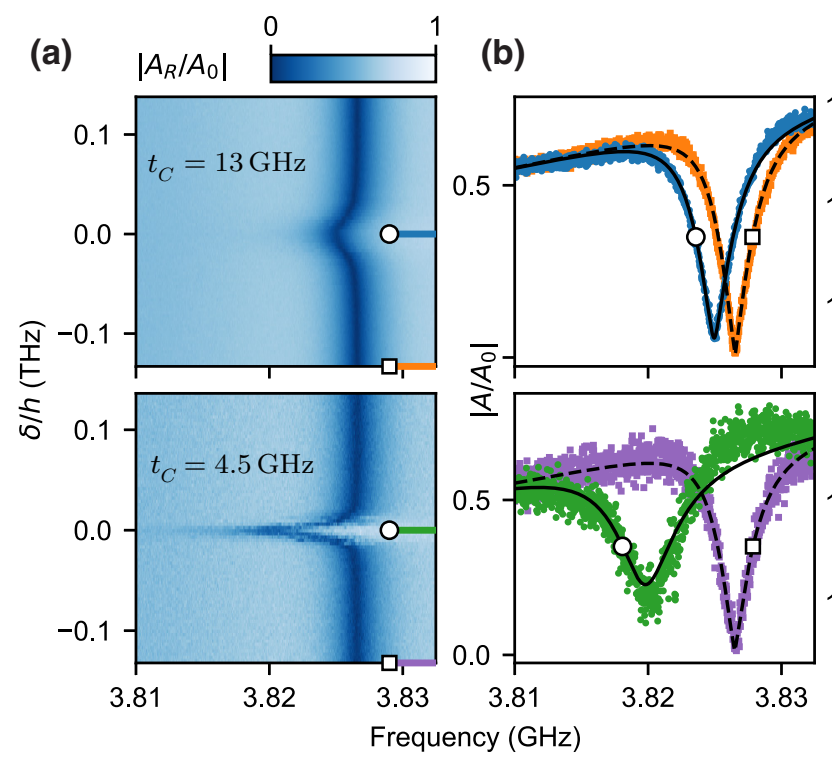

(c)

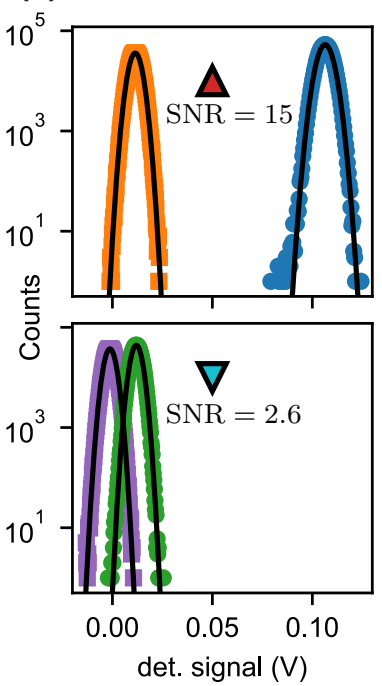

(d)

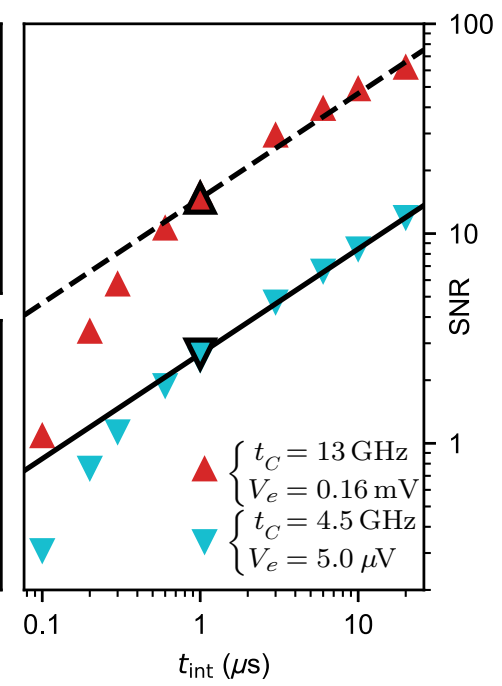

FIG. 5. Readout SNR. (a) Amplitude response as a function of detuning $\delta$ of the resonator coupled to the right dot for the two different tunnel coupling regimes. (b) Linecuts for Coulomb blockade (square marker) and on resonance (circle marker) together with fits to the theoretical model. (c) Histograms of the resonator responses in Coulomb blockade and charge degeneracy, with pulse length of $1 \mu \mathrm{s}$. Responses are acquired with a probe frequency tuned to resonance for the Coulomb blockade case, at approximately 3.826 GHz. (d) Attained SNR on the right dot's resonator, defined as $\Delta /(2 \sigma)$, as a function of measurement pulse length. Optimized with excitation voltage as a free parameter (red, up triangles) and optimized at fixed excitation voltage of $5 \mu \mathrm{V}$ (aqua, down triangles).

We define SNR as the change in signal between charge degeneracy and Coulomb blockade divided by the noise. To measure it, we perform a series of pulsed measurements of $I$ and $Q$ with a pulse time of $t_{\text {int }}$ at both Coulomb blockade and charge degeneracy, and show the obtained histograms for an integration time of $t_{\text {int }}=1 \mu \mathrm{s}$ in Fig. 5(c). These histograms are fit with a Gaussian to extract the separation between the Gaussian peaks $\Delta$ in the $I Q$ plane as well as their average standard deviation $\sigma$ representing the width. The SNR is given by $\Delta /(2 \sigma)$. More details are given in the Supplemental Material [34].

In Fig. 5(d) we plot the dependence of SNR on $t_{\text {int }}$, which approaches a square-root dependence for longer times. We attribute the discrepancy between attained SNR and a square-root dependence for pulse times shorter than $1 \mu$ s to the finite bandwidth of the resonators. For these pulse lengths, the resonator cannot reach a steadystate photon population, limiting the signal available for readout.

Next, we compare the observed SNR with expected theoretical limits. The change in signal at the feedline level $\Delta_{f}=\Delta / G_{\text {sys }}$ - with $G_{\text {sys }}$ the gain of the amplification chain in the system - can never exceed the total voltage swing in the feedline $V_{f}$. The fit to the data in Fig. 5(a), used to extract $t_{C}$, also provides a direct measurement of the ratio $\Delta_{f} / V_{f}=0.89$, close to the absolute maximum. In other words, the resonator is coupled near optimally for this tunnel coupling, such that its external coupling rate is nearly equal to the dispersive shift.
The achievable SNR is then set by $\Delta_{f}$ together with noise temperature, $T_{N}$, and readout time, $t_{\text {int }}$, as

$$
\mathrm{SNR}=\frac{\Delta_{f} \sqrt{t_{\text {int }}}}{2 \sqrt{Z k_{B} T_{N}}}
$$

where $Z=50 \Omega$ is the impedance of the feedline [34,61]. The SNR $\simeq 2.6$ found in Fig. 5(c) together with the readout time $t_{\text {int }}=1 \mu \mathrm{s}$ and the deduced approximate voltage swing in the feedline $V_{f}=0.15 \mu \mathrm{V}$ corresponds to a noise temperature estimate of $T_{N}=1 \mathrm{~K}$. Without the use of a TWPA, we expect that the noise temperature would increase to $T_{N} \approx 4 \mathrm{~K}$. To improve the $\mathrm{SNR}$, one can increase either the readout time or readout power in accordance with Eq. (5), as shown in Fig. 5(d). In practice, limits to these two parameters will be determined by the specific qubit implementation. Specifically, by optimizing the excitation voltage and tunnel coupling together, a SNR of 15 is achieved at $V_{e}=0.16 \mathrm{mV}$.

\section{CONCLUSIONS}

We show the characterization of an InAs nanowire multi-QD system using gigahertz-frequency sensing. Probing the finite frequency admittance of the nanowire allows us to infer the low-frequency conductance with good accuracy, even without calibration from dc measurements. Further, we show high-SNR dispersive sensing on timescales near the bandwidth limit set by the $Q$ factor 
of the resonators. Besides the use for qubit devices, we envision that fast multiplexed readout of quantum devices may be used for more complex sensing schemes. In particular, rapid simultaneous conduction of multiple local measurements could facilitate unique quantum transport experiments because they provide spatial information about tunneling processes. For example, probing two quantum dots at either end of a central charge island, tunneling events into the outer dots may be correlated $[62,63]$. We conclude that multiplexed SHF resonators may serve as a complete toolset for characterization and readout of semiconductor quantum devices, and present intriguing opportunities for developing high-speed quantum transport measurement schemes. Original data are available via the online data repository [64].

\section{ACKNOWLEDGMENTS}

We thank D. Bouman and J.D. Mensingh for nanowire deposition and A. Bargerbos for valuable comments on the manuscript. We further thank N.P. Alberts, O.W.B. Benningshof, R.N. Schouten, M.J. Tiggelman, and R.F.L. Vermeulen for valuable technical assistance. This work is supported by the Netherlands Organization for Scientific Research (NWO) and Microsoft.

[1] R. J. Schoelkopf, P. Wahlgren, A. A. Kozhevnikov, P. Delsing, and D. E. Prober, The radio-frequency singleelectron transistor (rf-set): A fast and ultrasensitive electrometer, Science 280, 1238 (1998).

[2] A. Blais, R.-S. Huang, A. Wallraff, S. M. Girvin, and R. J. Schoelkopf, Cavity quantum electrodynamics for superconducting electrical circuits: An architecture for quantum computation, Phys. Rev. A 69, 062320 (2004).

[3] A. Wallraff, D. I. Schuster, A. Blais, L. Frunzio, J. Majer, M. H. Devoret, S. M. Girvin, and R. J. Schoelkopf, Approaching Unit Visibility for Control of a Superconducting Qubit with Dispersive Readout, Phys. Rev. Lett. 95, 060501 (2005).

[4] R. Vijay, D. H. Slichter, and I. Siddiqi, Observation of Quantum Jumps in a Superconducting Artificial Atom, Phys. Rev. Lett. 106, 110502 (2011).

[5] T. Walter, P. Kurpiers, S. Gasparinetti, P. Magnard, A. Potočnik, Y. Salathé, M. Pechal, M. Mondal, M. Oppliger, C. Eichler, and A. Wallraff, Rapid High-Fidelity SingleShot Dispersive Readout of Superconducting Qubits, Phys. Rev. Appl. 7, 054020 (2017).

[6] J. Majer, J. M. Chow, J. M. Gambetta, J. Koch, B. R. Johnson, J. A. Schreier, L. Frunzio, D. I. Schuster, A. A. Houck, A. Wallraff, A. Blais, M. H. Devoret, S. M. Girvin, and R. J. Schoelkopf, Coupling superconducting qubits via a cavity bus, Nature 449, 443 (2007).

[7] M. A. Sillanpää, J. I. Park, and R. W. Simmonds, Coherent quantum state storage and transfer between two phase qubits via a resonant cavity, Nature 449, 438 (2007).
[8] P. Scarlino, D. J. van Woerkom, U. C. Mendes, J. V. Koski, A. J. Landig, C. K. Andersen, S. Gasparinetti, C. Reichl, W. Wegscheider, K. Ensslin, T. Ihn, A. Blais, and A. Wallraff, Coherent microwave-photon-mediated coupling between a semiconductor and a superconducting qubit, Nat. Commun. 10, 3011 (2019).

[9] F. Borjans, X. G. Croot, X. Mi, M. J. Gullans, and J. R. Petta, Resonant microwave-mediated interactions between distant electron spins, Nature 577, 195 (2019).

[10] M. Jerger, S. Poletto, P. Macha, U. Hübner, E. Il'ichev, and A. V. Ustinov, Frequency division multiplexing readout and simultaneous manipulation of an array of flux qubits, Appl. Phys. Lett. 101, 042604 (2012).

[11] E. Jeffrey, D. Sank, J. Mutus, T. White, J. Kelly, R. Barends, Y. Chen, Z. Chen, B. Chiaro, A. Dunsworth, A. Megrant, P. O'Malley, C. Neill, P. Roushan, A. Vainsencher, J. Wenner, A. Cleland, and J. M. Martinis, Fast Accurate State Measurement with Superconducting Qubits, Phys. Rev. Lett. 112, 190504 (2014).

[12] M. R. Delbecq, V. Schmitt, F. D. Parmentier, N. Roch, J. J. Viennot, G. Fève, B. Huard, C. Mora, A. Cottet, and T. Kontos, Coupling a Quantum dot, Fermionic Leads, and a Microwave Cavity on a Chip, Phys. Rev. Lett. 107, 256804 (2011)

[13] G. Puebla-Hellmann and A. Wallraff, Realization of gigahertz-frequency impedance matching circuits for nanoscale devices, Appl. Phys. Lett. 101, 053108 (2012).

[14] T. Hasler, M. Jung, V. Ranjan, G. Puebla-Hellmann, A. Wallraff, and C. Schönenberger, Shot Noise of a Quantum dot Measured with Gigahertz Impedance Matching, Phys. Rev. Appl. 4, 054002 (2015).

[15] J. Stehlik, Y.-Y. Liu, C. Quintana, C. Eichler, T. Hartke, and J. Petta, Fast Charge Sensing of a Cavity-Coupled Double Quantum dot Using a Josephson Parametric Amplifier, Phys. Rev. Appl. 4, 014018 (2015).

[16] V. Ranjan, G. Puebla-Hellmann, M. Jung, T. Hasler, A. Nunnenkamp, M. Muoth, C. Hierold, A. Wallraff, and C. Schönenberger, Clean carbon nanotubes coupled to superconducting impedance-matching circuits, Nat. Commun. 6, 7165 (2015).

[17] T. Frey, P. J. Leek, M. Beck, A. Blais, T. Ihn, K. Ensslin, and A. Wallraff, Dipole Coupling of a Double Quantum dot to a Microwave Resonator, Phys. Rev. Lett. 108, 046807 (2012).

[18] K. D. Petersson, L. W. McFaul, M. D. Schroer, M. Jung, J. M. Taylor, A. A. Houck, and J. R. Petta, Circuit quantum electrodynamics with a spin qubit, Nature 490, 380 (2012)

[19] X. Mi, J. V. Cady, D. M. Zajac, P. W. Deelman, and J. R. Petta, Strong coupling of a single electron in silicon to a microwave photon, Science 355, 156 (2016).

[20] A. Stockklauser, P. Scarlino, J. Koski, S. Gasparinetti, C. Andersen, C. Reichl, W. Wegscheider, T. Ihn, K. Ensslin, and A. Wallraff, Strong Coupling Cavity QED with Gate-Defined Double Quantum Dots Enabled by a High Impedance Resonator, Phys. Rev. X 7, 011030 (2017).

[21] L. E. Bruhat, T. Cubaynes, J. J. Viennot, M. C. Dartiailh, M. M. Desjardins, A. Cottet, and T. Kontos, Circuit QED with a quantum-dot charge qubit dressed by cooper pairs, Phys. Rev. B 98, 155313 (2018). 
[22] A. J. Landig, J. V. Koski, P. Scarlino, U. C. Mendes, A. Blais, C. Reichl, W. Wegscheider, A. Wallraff, K. Ensslin, and T. Ihn, Coherent spin-photon coupling using a resonant exchange qubit, Nature 560, 179 (2018).

[23] X. Mi, M. Benito, S. Putz, D. M. Zajac, J. M. Taylor, G. Burkard, and J. R. Petta, A coherent spin-photon interface in silicon, Nature 555, 599 (2018).

[24] N. Samkharadze, G. Zheng, N. Kalhor, D. Brousse, A. Sammak, U. C. Mendes, A. Blais, G. Scappucci, and L. M. K. Vandersypen, Strong spin-photon coupling in silicon, Science 359, 1123 (2018).

[25] G. Zheng, N. Samkharadze, M. L. Noordam, N. Kalhor, D. Brousse, A. Sammak, G. Scappucci, and L. M. K. Vandersypen, Rapid gate-based spin read-out in silicon using an on-chip resonator, Nat. Nanotechnol. 14, 742 (2019).

[26] J. V. Koski, A. J. Landig, M. Russ, J. C. Abadillo-Uriel, P. Scarlino, B. Kratochwil, C. Reichl, W. Wegscheider, G. Burkard, M. Friesen, S. N. Coppersmith, A. Wallraff, K. Ensslin, and T. Ihn, Strong photon coupling to the quadrupole moment of an electron in a solid-state qubit, Nat. Phys. 16, 642 (2020).

[27] M.-C. Harabula, T. Hasler, G. Fülöp, M. Jung, V. Ranjan, and C. Schönenberger, Measuring a Quantum dot with an Impedance-Matching On-Chip Superconducting LC Resonator at Gigahertz Frequencies, Phys. Rev. Appl. 8, 054006 (2017).

[28] N. Holman, D. Rosenberg, D. Yost, J. L. Yoder, R. Das, W. D. Oliver, R. McDermott, and M. A. Eriksson, Dispersive measurement of a semiconductor double quantum dot via $3 \mathrm{~d}$ integration of a high-impedance tin resonator, arXiv:2011.08759 [cond-mat.mes-hall] (2020).

[29] A. Ruffino, T.-Y. Yang, J. Michniewicz, Y. Peng, E. Charbon, and M. F. Gonzalez-Zalba, Integrated multiplexed microwave readout of silicon quantum dots in a cryogenic cmos chip, arXiv:2101.08295 [quant-ph] (2021).

[30] V. D. Maman, M. Gonzalez-Zalba, and A. Pályi, Charge Noise and Overdrive Errors in Dispersive Readout of Charge, Spin, and Majorana Qubits, Phys. Rev. Appl. 14, 064024 (2020).

[31] I. Ahmed, J. A. Haigh, S. Schaal, S. Barraud, Y. Zhu, C. min Lee, M. Amado, J. W. A. Robinson, A. Rossi, J. J. L. Morton, and M. F. Gonzalez-Zalba, Radio-Frequency Capacitive Gate-Based Sensing, Phys. Rev. Appl. 10, 014018 (2018).

[32] C. Macklin, K. O’Brien, D. Hover, M. E. Schwartz, V. Bolkhovsky, X. Zhang, W. D. Oliver, and I. Siddiqi, A near-quantum-limited josephson traveling-wave parametric amplifier, Science 350, 307 (2015).

[33] J. Kroll, F. Borsoi, K. van der Enden, W. Uilhoorn, D. de Jong, M. Quintero-Pérez, D. van Woerkom, A. Bruno, S. Plissard, D. Car, E. Bakkers, M. Cassidy, and L. Kouwenhoven, Magnetic-Field-Resilient Superconducting Coplanar-Waveguide Resonators for Hybrid Circuit Quantum Electrodynamics Experiments, Phys. Rev. Appl. 11, 064053 (2019).

[34] See Supplemental Material at http://link.aps.org/supple mental/10.1103/PhysRevApplied.16.014007 for further details of the experimental setup, derivations of the equations in this article, and additional figures of the experimental data.
[35] S. J. Chorley, J. Wabnig, Z. V. Penfold-Fitch, K. D. Petersson, J. Frake, C. G. Smith, and M. R. Buitelaar, Measuring the Complex Admittance of a Carbon Nanotube Double Quantum dot, Phys. Rev. Lett. 108, 036802 (2012).

[36] D. Razmadze, D. Sabonis, F. K. Malinowski, G. C. Ménard, S. Pauka, H. Nguyen, D. M. van Zanten, E. C. O'Farrell, J. Suter, P. Krogstrup, F. Kuemmeth, and C. M. Marcus, Radio-Frequency Methods for Majorana-Based Quantum Devices: Fast Charge Sensing and Phase-Diagram Mapping, Phys. Rev. Appl. 11, 064011 (2019).

[37] M. S. Khalil, M. J. A. Stoutimore, F. C. Wellstood, and K. D. Osborn, An analysis method for asymmetric resonator transmission applied to superconducting devices, J. Appl. Phys. 111, 054510 (2012).

[38] S. Probst, F. B. Song, P. A. Bushev, A. V. Ustinov, and M. Weides, Efficient and robust analysis of complex scattering data under noise in microwave resonators, Rev. Sci. Instrum. 86, 024706 (2015).

[39] H. Guan, M. Dai, Q. He, J. Hu, P. Ouyang, Y. Wang, L. F. Wei, and J. Gao, Network modeling of non-ideal superconducting resonator circuits, Supercond. Sci. Technol. 33, 075004 (2020).

[40] W. G. van der Wiel, S. D. Franceschi, J. M. Elzerman, T. Fujisawa, S. Tarucha, and L. P. Kouwenhoven, Electron transport through double quantum dots, Rev. Mod. Phys. 75, 1 (2002).

[41] J. Gabelli, G. Fève, J.-M. Berroir, B. Plaçais, A. Cavanna, B. Etienne, Y. Jin, and D. C. Glattli, Violation of kirchhoff's laws for a coherent rc circuit, Science 313, 499 (2006).

[42] M. Jung, M. D. Schroer, K. D. Petersson, and J. R. Petta, Radio frequency charge sensing in InAs nanowire double quantum dots, Appl. Phys. Lett. 100, 253508 (2012)

[43] N. Ares, F. Schupp, A. Mavalankar, G. Rogers, J. Griffiths, G. Jones, I. Farrer, D. Ritchie, C. Smith, A. Cottet, G. Briggs, and E. Laird, Sensitive Radio-Frequency Measurements of a Quantum dot by Tuning to Perfect Impedance Matching, Phys. Rev. Appl. 5, 034011 (2016).

[44] K. D. Petersson, C. G. Smith, D. Anderson, P. Atkinson, G. A. C. Jones, and D. A. Ritchie, Charge and spin state readout of a double quantum dot coupled to a resonator, Nano Lett. 10, 2789 (2010).

[45] J. I. Colless, A. C. Mahoney, J. M. Hornibrook, A. C. Doherty, H. Lu, A. C. Gossard, and D. J. Reilly, Dispersive Readout of a Few-Electron Double Quantum dot with Fast rf Gate Sensors, Phys. Rev. Lett. 110, 046805 (2013).

[46] A. C. Betz, R. Wacquez, M. Vinet, X. Jehl, A. L. Saraiva, M. Sanquer, A. J. Ferguson, and M. F. Gonzalez-Zalba, Dispersively detected pauli spin-blockade in a silicon nanowire field-effect transistor, Nano Lett. 15, 4622 (2015).

[47] A. A. Esmail, A. J. Ferguson, and N. J. Lambert, Cooper pair tunnelling and quasiparticle poisoning in a galvanically isolated superconducting double dot, Appl. Phys. Lett. 111, 252602 (2017).

[48] P. Pakkiam, A. Timofeev, M. House, M. Hogg, T. Kobayashi, M. Koch, S. Rogge, and M. Simmons, SingleShot Single-Gate rf Spin Readout in Silicon, Phys. Rev. X 8, 041032 (2018). 
[49] M. Urdampilleta, D. J. Niegemann, E. Chanrion, B. Jadot, C. Spence, P.-A. Mortemousque, C. Bäuerle, L. Hutin, B. Bertrand, S. Barraud, R. Maurand, M. Sanquer, X. Jehl, S. D. Franceschi, M. Vinet, and T. Meunier, Gatebased high fidelity spin readout in a CMOS device, Nat. Nanotechnol. 14, 737 (2019).

[50] A. West, B. Hensen, A. Jouan, T. Tanttu, C.-H. Yang, A. Rossi, M. F. Gonzalez-Zalba, F. Hudson, A. Morello, D. J. Reilly, and A. S. Dzurak, Gate-based single-shot readout of spins in silicon, Nat. Nanotechnol. 14, 437 (2019).

[51] D. de Jong, J. van Veen, L. Binci, A. Singh, P. Krogstrup, L. P. Kouwenhoven, W. Pfaff, and J. D. Watson, Rapid Detection of Coherent Tunneling in an InAs Nanowire Quantum dot through Dispersive Gate Sensing, Phys. Rev. Appl. 11, 044061 (2019).

[52] D. Sabonis, E. C. T. O'Farrell, D. Razmadze, D. M. T. van Zanten, J. Suter, P. Krogstrup, and C. M. Marcus, Dispersive sensing in hybrid InAs/al nanowires, Appl. Phys. Lett. 115, 102601 (2019).

[53] A. Crippa, R. Ezzouch, A. Aprá, A. Amisse, R. Laviéville, L. Hutin, B. Bertrand, M. Vinet, M. Urdampilleta, T. Meunier, M. Sanquer, X. Jehl, R. Maurand, and S. D. Franceschi, Gate-reflectometry dispersive readout and coherent control of a spin qubit in silicon, Nat. Commun. 10, 2776 (2019).

[54] M. Esterli, R. M. Otxoa, and M. F. Gonzalez-Zalba, Smallsignal equivalent circuit for double quantum dots at lowfrequencies, Appl. Phys. Lett. 114, 253505 (2019).

[55] S. Park, C. Metzger, L. Tosi, M. Goffman, C. Urbina, H. Pothier, and A. L. Yeyati, From Adiabatic to Dispersive Readout of Quantum Circuits, Phys. Rev. Lett. 125, 077701 (2020).

[56] F. J. Schupp, F. Vigneau, Y. Wen, A. Mavalankar, J. Griffiths, G. A. C. Jones, I. Farrer, D. A. Ritchie, C. G. Smith, L. C. Camenzind, L. Yu, D. M. Zumbühl, G. A. D. Briggs,
N. Ares, and E. A. Laird, Sensitive radiofrequency readout of quantum dots using an ultra-low-noise SQUID amplifier, J. Appl. Phys. 127, 244503 (2020).

[57] J. M. Hornibrook, J. I. Colless, A. C. Mahoney, X. G. Croot, S. Blanvillain, H. Lu, A. C. Gossard, and D. J. Reilly, Frequency multiplexing for readout of spin qubits, Appl. Phys. Lett. 104, 103108 (2014).

[58] S. Plugge, A. Rasmussen, R. Egger, and K. Flensberg, Majorana box qubits, New J. Phys. 19, 012001 (2017).

[59] T. Karzig, C. Knapp, R. M. Lutchyn, P. Bonderson, M. B. Hastings, C. Nayak, J. Alicea, K. Flensberg, S. Plugge, Y. Oreg, C. M. Marcus, and M. H. Freedman, Scalable designs for quasiparticle-poisoning-protected topological quantum computation with majorana zero modes, Phys. Rev. B 95, 235305 (2017).

[60] T. B. Smith, M. C. Cassidy, D. J. Reilly, S. D. Bartlett, and A. L. Grimsmo, Dispersive readout of majorana qubits, PRX Quantum 1, 020313 (2020).

[61] D. Pozar, Microwave Engineering (Wiley, Hoboken, NJ, 2011), 4th ed.

[62] Z. Tan, D. Cox, T. Nieminen, P. Lähteenmäki, D. Golubev, G. Lesovik, and P. Hakonen, Cooper Pair Splitting by Means of Graphene Quantum Dots, Phys. Rev. Lett. 114, 096602 (2015).

[63] G. Ménard, G. Anselmetti, E. Martinez, D. Puglia, F. Malinowski, J. Lee, S. Choi, M. Pendharkar, C. Palmstrøm, K. Flensberg, C. Marcus, L. Casparis, and A. Higginbotham, Conductance-Matrix Symmetries of a Three-Terminal Hybrid Device, Phys. Rev. Lett. 124, 036802 (2020).

[64] D. de Jong, C. Prosko, D. M. A. Waardenburg, L. Han, F. K. Malinowski, P. Krogstrup, L. P. Kouwenhoven, J. V. Koski, and W. Pfaff, Data for: "Rapid microwave-only characterization and readout of quantum dots using multiplexed gigahertz-frequency resonators," https://doi.org/10.5281/zenodo.5036156 (2021). 